\title{
Foreground-Background Segmentation Revealed during Natural Image Viewing
}

\author{
DPaolo Papale, ${ }^{1}$ (D)Andrea Leo, ${ }^{1}$ DLuca Cecchetti, ${ }^{1}$ Giacomo Handjaras, ${ }^{1}$ Kendrick N. Kay, ${ }^{2}$ Pietro \\ Pietrini, ${ }^{1}$ and ${ }^{\mathbb{D}}$ Emiliano Ricciardi ${ }^{1 *}$
}

DOI:http://dx.doi.org/10.1523/ENEURO.0075-18.2018

${ }^{1}$ Molecular Mind Lab, IMT School for Advanced Studies Lucca, Lucca, 55100 Italy and ${ }^{2}$ Center for Magnetic
Resonance Research, Department of Radiology, University of Minnesota, Twin Cities, Minneapolis, MN, 55455

\begin{abstract}
One of the major challenges in visual neuroscience is represented by foreground-background segmentation. Data from nonhuman primates show that segmentation leads to two distinct, but associated processes: the enhancement of neural activity during figure processing (i.e., foreground enhancement) and the suppression of background-related activity (i.e., background suppression). To study foreground-background segmentation in ecological conditions, we introduce a novel method based on parametric modulation of low-level image properties followed by application of simple computational image-processing models. By correlating the outcome of this procedure with human fMRI activity, measured during passive viewing of 334 natural images, we produced easily interpretable "correlation images" from visual populations. Results show evidence of foreground enhancement in all tested regions, from V1 to lateral occipital complex (LOC), while background suppression occurs in V4 and LOC only. Correlation images derived from V4 and LOC revealed a preserved spatial resolution of foreground textures, indicating a richer representation of the salient part of natural images, rather than a simplistic model of object shape. Our results indicate that scene segmentation occurs during natural viewing, even when individuals are not required to perform any particular task.
\end{abstract}

Key words: figure-ground; fMRl; human; natural scenes; visual cortex; visual perception

\section{Significance Statement}

Foreground-background segmentation has been considered critical to form discrete object representations from continuous sensory percepts. We developed a pre-filtering approach which overcame typical limitations in modeling brain responses to complex stimuli and could be generalized to related processes. Our findings provide novel support to the hypothesis that foreground-background segmentation of natural scenes occurs during passive perception, sustained by the distributed activity of multiple areas across the visual processing stream. Specifically, while foreground information is enhanced along the entire visual pathway, V4 and lateral occipital complex (LOC) show a background suppression effect, though retaining texture information from the foreground. Our observations challenge the idea that these regions of the visual system may primarily encode simple object representations based on silhouette or shape features only.

\section{Introduction}

In the scientific journey toward a satisfying understanding of the human visual system, scene segmentation represents a central problem "for which no theoretical

Received February 19, 2018; accepted May 15, 2018; First published May 30, 2018.

The authors declare no competing financial interests.

Author contributions: P.Pa., A.L., L.C., G.H., K.N.K., P.Pi., and E.R. designed research; K.N.K. performed research; P.Pa. analyzed data; P.Pa., A.L., L.C., G.H., K.N.K., P.Pi., and E.R. wrote the paper. solution exists" (Wu et al., 2006). Segmentation into foreground and background is crucial to make sense of the surrounding visual environment, and its pivotal role as an initial step of visual content identification has long been

Acknowledgements: This work was supported by Italian Ministry for Education, University and Research Grants PRIN 2015WXAXJF and PRIN 2015AR52F9.

Correspondence should be addressed to Emiliano Ricciardi, IMT School for Advanced Studies Lucca, Piazza San Francesco 19, Lucca, 55100, Italy. E-mail: emiliano.ricciardi@imtlucca.it. 
theorized (Biederman, 1987). Indeed, according to Fowlkes et al. (2007), humans can produce consistent segmentations of natural images. However, although more recent approaches based on deep convolutional networks produced promising results (He et al., 2017), both the computational and neurophysiological processes that underlie scene segmentation are still a matter of debate.

To date, numerous studies found evidence of texture segmentation and figure-ground organization in the early visual cortex of nonhuman primates (Lamme, 1995; Lee et al., 1998; Poort et al., 2012; Self et al., 2013) and humans (Kastner et al., 2000; Scholte et al., 2008; Kok and de Lange, 2014). It has been showed that the identification of salient visual attributes arises from a regionfilling mechanism that targets neural populations mapping relevant points in space (Roelfsema, 2006). In particular, a recent study on monkeys attending artificial stimuli revealed an early enhancement of V1 and V4 neurons when their receptive fields covered the foreground and a later response suppression when their receptive fields were located in the stimulus background (Poort et al., 2016), extending results from a previous study (Lamme et al., 1999). Thus, the primate brain groups together image elements which belong to the figure, showing an enhanced activity for the foreground and a concurrent suppression of the background.

However, from an experimental viewpoint, the role of figure-ground segmentation has primarily been demonstrated by means of non-ecological stimuli (e.g., binary figures, random dots, oriented line segments and textures). It should be noted that previous reports demonstrated how models of brain responses to artificial stimuli are suboptimal in predicting responses to natural images (David et al., 2004; Felsen and Dan, 2005). Although two recent studies investigated border-ownership in monkeys with both artificial and natural stimuli (Hesse and Tsao, 2016; Williford and von der Heydt, 2016), a proof of the occurrence of foreground-background segmentation in the human brain during visual processing of naturalistic stimuli (e.g., natural images and movies) is still lacking. This pushes toward the development of novel methods specifically designed for testing segmentation in ecological conditions.

In light of this, we investigated foreground enhancement and background suppression, as specific processes involved in scene segmentation during passive viewing of natural images. We used fMRI data, previously published by Kay et al. (2008), to study brain activity patterns from seven visual regions of interest (ROIs): V1, V2, V3, V3A, $\mathrm{V} 3 \mathrm{~B}, \mathrm{~V} 4$, and lateral occipital complex (LOC) in response to 334 natural images, whose "ground-truth" segmented counterparts have been included in the Berkeley Segmentation Dataset (BSD; Arbeláez et al., 2011).

To this aim, we developed a novel pre-filtering modeling approach to study brain responses to complex, natural

DOI:http://dx.doi.org/10.1523/ENEURO.0075-18.2018 Copyright (C) 2018 Papale et al.

This is an open-access article distributed under the terms of the Creative Commons Attribution 4.0 International license, which permits unrestricted use, distribution and reproduction in any medium provided that the original work is properly attributed. images without relying on explicit models of scene segmentation and adopting a validated and biologically plausible description of activity in visual cortices. Our method is similar to other approaches where explicit computations are performed on representational features, rather than on the original stimuli (Naselaris et al., 2011). For instance, these methods have been recently used to investigate semantic representation (Huth et al., 2012; Handjaras et al., 2017) or boundary and surface-related features (Lescroart et al., 2016). However, as opposed to the standard modeling framework, according to which alternative models are computed from the stimuli to predict brain responses, here, low-level features of the stimuli are parametrically modulated and simple descriptors of each filtered image (i.e., edges position, size and orientation) are aggregated in a fixed model (Fig. 1). The correspondence between the fixed model and fMRI representational geometry related to intact images, was then evaluated using representational similarity analysis (RSA; Kriegeskorte et al., 2008). Notably, this approach can also be exploited to obtain highly informative "correlation images" representing the putative computations of different brain regions and may be generalized to investigate different phenomena in visual neuroscience.

\section{Materials and Methods}

To assess differences between cortical processes involved in foreground-background segmentation, we employed a low-level description of images (edge position, size and orientation), defined by a weighted sum of the representational dissimilarity matrices (RDMs) of four well-known computational models (Fig. 2D). These models are based on simple features (edge position, size and orientation), whose physiologic counterparts are well known (Marr, 1982). The model was kept constant while the images were parametrically filtered and iteratively correlated with representational measures of brain activity through RSA. For each ROI, this pre-filtering modeling approach led to a pictorial and easily interpretable representation of the optimal features (contrast and spatial frequencies) of foreground and background of natural images (i.e., correlation images). The analytical pipeline is schematized in Figure 2.

\section{Stimuli and behavioral segmentation of foreground and background}

We selected from the 1870 images used by Kay et al. (2008) a subsample of 334 pictorial stimuli which are also represented in the BSD 500 (Arbeláez et al., 2011). For each BSD image, five to seven subjects manually performed an individual ground-truth segmentation, which is provided by the authors of the dataset (http://www.eecs.berkeley.edu/ Research/Projects/CS/vision/grouping/resources.html). Although figure-ground judgment is rather stable across subjects (Fowlkes et al., 2007), we selected the largest patch, manually labeled as foreground, among the behavioral segmentations, to build a foreground binary mask. For each image, this mask was then down-sampled and applied to the original stimulus to isolate the foreground and the background pixels (Kay et al., 2008). 
A

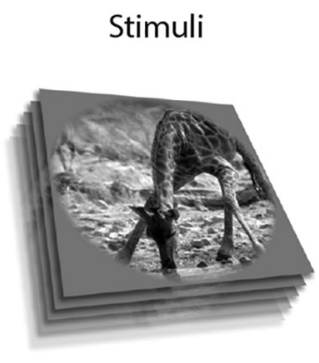

B

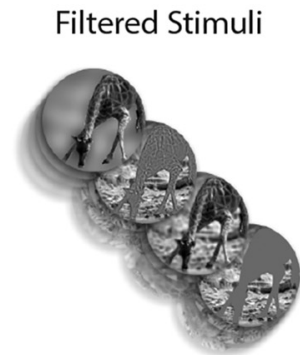

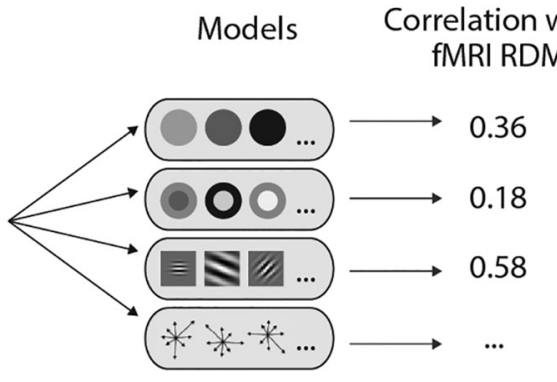
fMRI RDM
Result

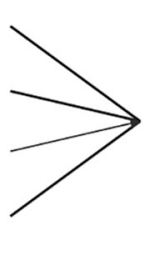

\section{Best model}

Model

Correlation with
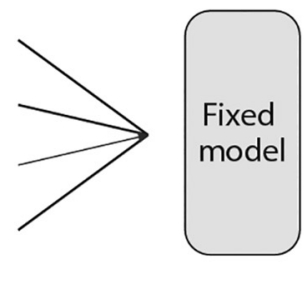
fMRI RDM

Result

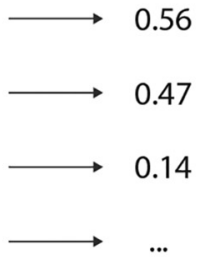

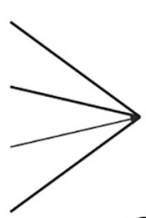

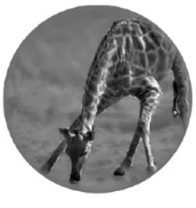

Correlation image

Figure 1. Comparing the standard modeling approach and the pre-filtering modeling approach. $\boldsymbol{A}$, In the standard modeling pipeline, different models are compared. After extracting features from the stimuli, competing feature vectors can be used to predict brain activity in an encoding procedure, whereas their dissimilarities can be used in a RSA. Finally, the model that better predicts brain responses is discussed. $\boldsymbol{B}$, In our pre-filtering modeling approach, different filtered versions of the original stimuli are compared. Various biologically plausible filtering procedures are applied to the stimuli before compute a unique feature space according to a given fixed and easily interpretable model. In our approach, a single model is employed and the step showing the highest correlation with brain activity (or representational geometry) of each filtering procedure is used to build a post hoc correlation image. While the standard modeling approach is theoretically more advantageous, as its output is a fully computable model of brain activity, it cannot be applied when reliable explicit models of perceptual processes do not exist yet, as in the case of scene segmentation. Alternative attempts to reconstruct visual stimuli from brain activity have been previously reported using multivariate techniques (Stanley et al., 1999; Thirion et al., 2006; Miyawaki et al., 2008; Nishimoto et al., 2011).

\section{fMRI data}

The $\mathrm{fMRI}$ data used in this study are publicly available at http://crcns.org/data-sets/vc/vim-1 (Kay et al., 2011). Two subjects (males, age: 33 and 25) were acquired using the following MRI parameters: 4T INOVA MR, matrix size $64 \times 64$, TR 1 s, TE $28 \mathrm{~ms}$, flip angle $20^{\circ}$, spatial resolution $2 \times 2 \times 2.5 \mathrm{~mm}^{3}$. For each subject five scanning sessions (seven runs each) were performed on five separate days. The stimuli were 1870 greyscale natural images with diameter $20^{\circ}$ (500 pixels), embedded in a gray background, and were presented for $1 \mathrm{~s}$, flickering at $5 \mathrm{~Hz}$, with an ISI of $3 \mathrm{~s}$. Subjects were asked to fixate a central white square of $0.2^{\circ}$ (4 pixels). Seven visual ROls (V1, V2, V3, V3A, V3B, V4, and LOC) were defined and brain activity patterns related to stimulus presentation was extracted from these regions. For additional details on pre-processing, retinotopic mapping and ROls localization, refer to Kay et al. (2008).

\section{Computational models}

In accordance with a previous fMRI study that, to the best of our knowledge, has tested the highest number of computational models, we selected four untrained models: two showing highest correlations with brain activity patterns in early visual areas, and the others, showing highest correlations with LOC (Khaligh-Razavi and Kriegeskorte, 2014). All these models are based on biologically inspired features, such as Gabor filters and image gradient and comprise:
GIST (Oliva and Torralba, 2001), Dense SIFT (Lazebnik et al., 2006), Pyramid Histograms of Gradients (PHOG; Bosch et al., 2007), and Local Binary Patterns (LBP; Ojala et al., 2001). For an exhaustive description of the four models, and links to MATLAB codes, see Khaligh-Razavi (2014) and Khaligh-Razavi and Kriegeskorte (2014). Our model choice was also motivated by the fact that the stimuli were grayscale and had a fixed circular aperture. Thus, we excluded descriptions based on color or silhouette information, as well as pretrained convolutional neural networks which are biased toward the global shape of the image (Kubilius et al., 2016).

\section{RSA}

For each filtered image, we collected feature vectors from the four computational models (PHOG, GIST, LBP, and Dense SIFT), and RDMs were then obtained (1 minus the Pearson correlation metric). These four RDMs were normalized in a range between 0 and 1 and combined to obtain the fixed biologically plausible model of the stimuli (for a graphical representation of the process, see Fig. $2 D$ ). The four model RDMs were combined through a weighted sum, based on an estimation of their correlation with the representational model of brain activity. Single subject RDMs were similarly computed using fMRI activity patterns for each of the seven ROls, and then averaged across the two subjects. We used Spearman's $\rho$ to assess the correlation between the RDM from each step of the 
A behavioral segmentations

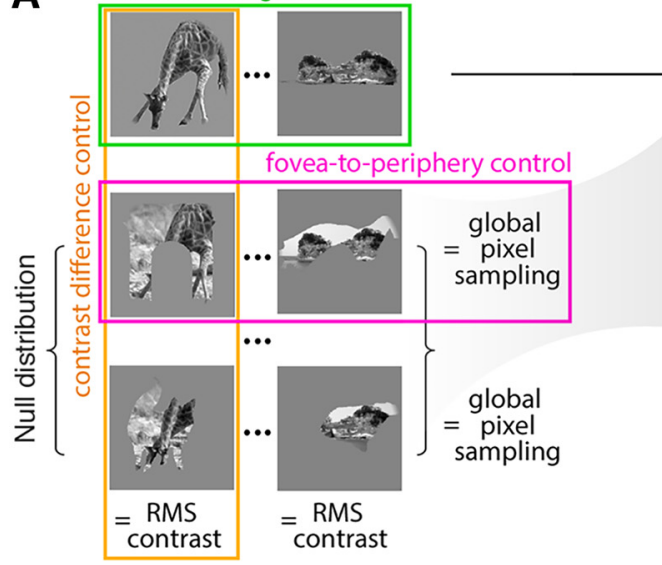

B

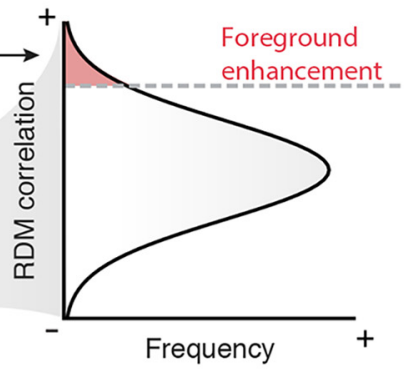

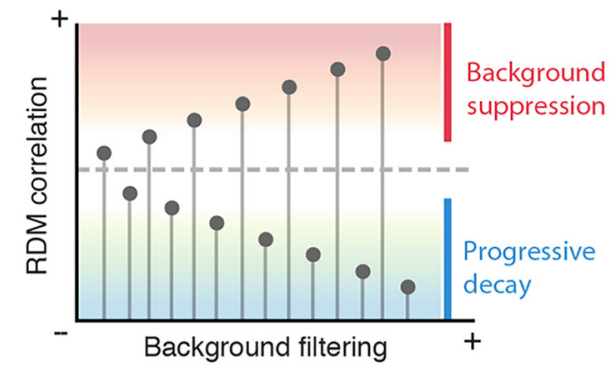

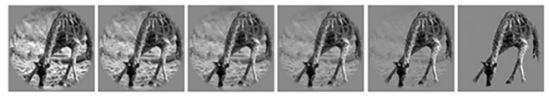

C

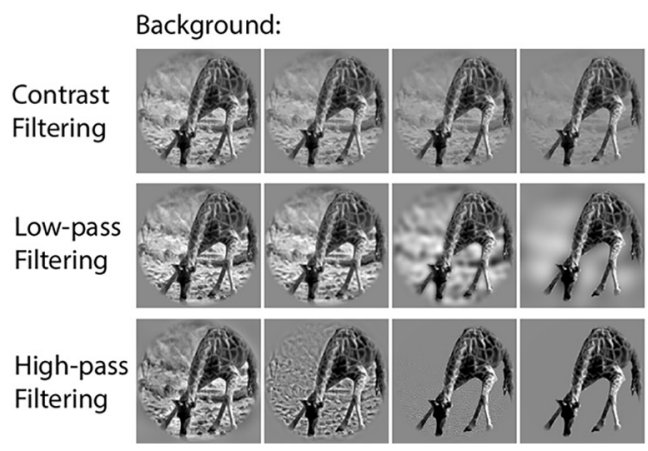

D

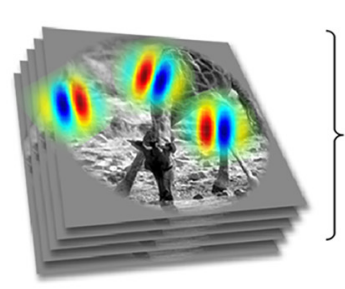

Extract features

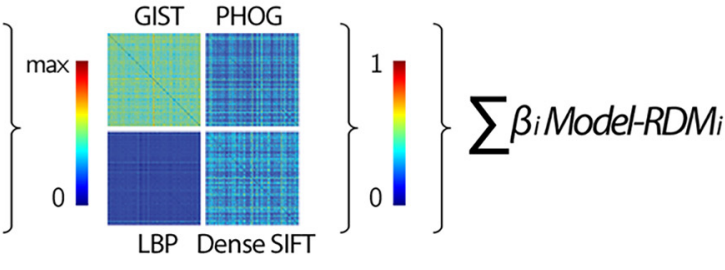

Compute RDMs

Normalize RDMs

Combine RDMs

Figure 2. Analytical pipeline. A, Foreground enhancement test: the set of segmented stimuli is tested against a null distribution of 1000 permutations. Each permutation is built by randomly shuffling the 334 behavioral foreground masks and matching the RMS contrast of the behaviorally segmented counterpart. This analysis controls for size, location, and contrast of the foreground when testing whether behavioral segmentations explain each ROI RDM better than chance. $\boldsymbol{B}$, Background suppression test: the correlation between brain RDMs and each step of the background filtering procedure is tested against the correlation determined by the intact stimuli. While information is filtered out, correlation can increase or decrease, depending on the sensitivity for background related information in each ROI. A progressive decay indicates that a region actually processes the background, while a significant increase suggests that background is suppressed. $\boldsymbol{C}$, Filtering steps for the contrast or spatial frequencies filtering. $\boldsymbol{D}$, From left to right: features for each model were extracted from the stimuli; the dissimilarity (1-Pearson's $r$ ) between each stimulus pair was computed and aggregated in four RDMs; the obtained RDMs were normalized in a 0-1 range; finally, the four RDMs were linearly combined in the fixed model, which was then correlated to the fMRI RDM obtained from each ROI.

image filtering procedures and the RDM of each brain ROI. To obtain unbiased estimations of the correlation between models and fMRI, a 5-fold cross-validation procedure based on a weighted sum of the models was developed: model weights were first estimated trough linear regression on a portion (80\%) of the RDMs, and the correlation with $\mathrm{fMRI}$ data were then computed based on the remainder of the RDMs (20\%). The correlation values derived from this procedure were averaged across the five folds, to obtain a unique estimate of the similarity between image features and brain activity. This analysis was performed independently in each of the seven ROls, and the SE for each correlation value was estimated with bootstrapping of the stimuli - 1000 iterations (Efron and Tibshirani, 1993).

In addition, as each ROI may show a distinct signal-tonoise ratio, we computed a noise estimation by correlating the brain RDMs extracted from the two subjects. This procedure allows for qualitative comparison between dif- ferent ROls and could help in estimate how well each model explains fMRI RDMs given the noise in the data.

\section{Foreground enhancement testing}

A permutation test was performed to statistically assess the enhancement of the information retained in the behavioral segmented foreground. In this test both the "fovea-to-periphery" bias that characterizes natural images, and possible differences in contrast between foreground and background were controlled (Fig. 2A). For each iteration, the 334 foreground masks were shuffled and a random foreground segmentation was associated to each stimulus. The root mean square (RMS) contrast of each obtained segmented image was matched to that of the behaviorally segmented counterpart. Of note, this set of randomly-segmented images had the same distribution of masked portions of the visual field as the one from the behavioral segmentation, so the same amount of information was isolated at each permutation step. This procedure was repeated 1000 times, to build a null distribution 
of alternative segmentations: four examples of random segmentation are shown in Figure $2 A$. For each permutation step, features were extracted from each randomly segmented image and RSA was performed using the procedure described above.

\section{Parametric filtering procedures}

To investigate differential processing of foreground and background in the visual system, we employed three different filtering procedures (contrast; through alpha channel modulation; low- and high-pass filtering of spatial frequencies) applied parametrically (four steps each) to the foreground or the background. For each filtering procedure, the four manipulated images are represented in Figure $2 C$. For low- and high-pass filtering, we employed a Butterworth filter (5th order), linearly sampling from a log-transformed distribution of frequencies ranging from 0.05 to 25 cycle per degree, while keeping the RMS contrast fixed.

\section{Background suppression testing}

To test background suppression, we performed a twotailed permutation test. In each ROI, we computed the difference between the correlation of the intact version of the stimuli and each step of the background filtering procedures (Fig. 2B). Afterward, a permutation test $(10,000$ iterations) was performed by random sampling two groups from the bootstrap distributions, obtaining a null distribution of correlation differences. Reported results are Bonferroni corrected (for the 13 comparisons in each ROI).

\section{Correlation images}

For each ROI, the effects of the filtering procedures were combined, to build correlation images. To this aim we used the filtering step with the highest correlation between the fixed model and RDMs from fMRI data, for foreground and background respectively. In detail, we averaged the best images for the low- and high-pass filters and multiplied each pixel for the preferred alphachannel value (contrast).

\section{Significance testing}

To assess the statistical significance of the correlations obtained with RSA in all the above-mentioned filtering procedures, we built a robust $\mathrm{ROI}$-specific permutation test (1000 iterations), by randomly sampling voxels of the occipital lobe not located in any of the seven ROls. We labeled these voxels as "control-voxels." This procedure has the advantage to be resilient to biases in fMRI data (Schreiber and Krekelberg, 2013), instead of simply taking into account the distribution of the RDM values, as in Khaligh-Razavi and Kriegeskorte (2014). In addition, the procedure that we developed is also useful to control for the effects related to number of voxels and to the signalto-noise ratio of each $\mathrm{ROI}$.

First, for each ROI we computed the SE of the ROIspecific noise estimation with bootstrap resampling of the stimuli (1000 iterations). Second, a number of control voxels equal to the number of voxels was randomly selected within each ROI, and the activity of these control voxels in response to the stimuli were used to build a null RDM. Third, the correlation between the null RDMs of the two subjects was computed. However, since we aimed at matching the signal-to-noise ratio of the null distribution to that of each ROI, the null RDM was counted as a valid permutation only if the single subject RDMs correlated to each other within a specific range (i.e., ROI-specific noise estimation $\pm \mathrm{SE}$ ). Finally, for each step of the filtering procedures, each of the $1000 \mathrm{ROI}$-specific null RDMs were correlated with the fixed model RDM to obtain a null distribution of $1000 \rho$ values. A one-tailed rank test was used to assess the significance of the $\rho$ of the fixed model with brain RDMs. For each ROI, we controlled for multiple comparisons (27 tests), through Bonferroni correction.

\section{Code accessibility}

All analyses have been implemented in MATLAB (MathWorks Inc.) using in-house developed code (available at https://bit.ly/2rC27hY). All code is also available as Extended Data 1.

\section{Results}

Foreground enhancement and background suppression can be tested in ecological conditions following a simple argument: when attempting to predict brain activity of a visual ROI with a specific model, the goodnessof-fit depends on the model inputs, e.g., the spatial information provided. Thus, the correlation between filtered images and fMRI representational patterns evoked by their intact counterpart can be used to verify specific hypotheses on visual processing (Fig. 2). In this study, we posit that evidence of preferential processing (i.e., enhancement) should depend on the shape of the foreground instead of the size, the location or the contrast of the segmented region processed through the model. In this regard, a random sampling procedure of foreground segmentations across stimuli would offer a proper choice to account for all these aspects, ultimately testing whether behavioral segmentations provide a better prior for enhancement. On the other hand, background filtering can lead either to a decay or an increase in correlation with brain representational patterns. The former indicates that background-related information is, at least to some extent, processed, whereas the latter denotes that background information is suppressed, since embedding it in the model is not different from adding noise.

\section{Comparison of intact and behaviorally segmented images}

The correlation between RDMs computed using the fMRI patterns from each of the seven visual ROls and three descriptions of the stimuli (intact, isolated background, and isolated foreground) were tested (Fig. 3; Table 1). Results show significant correlations $(p<0.05$ Bonferroni corrected) between the intact description of images and fMRI RDMs in V1, V2, and V3. The segmented foreground RDM shows a significant correlation in V2, V4, and LOC, while the segmented background achieves significant correlations in V1 and V2 only. Of note, the correlation yielded by one of the descriptions approaches the ROI-specific SNR estimation (i.e., the maximum reachable 


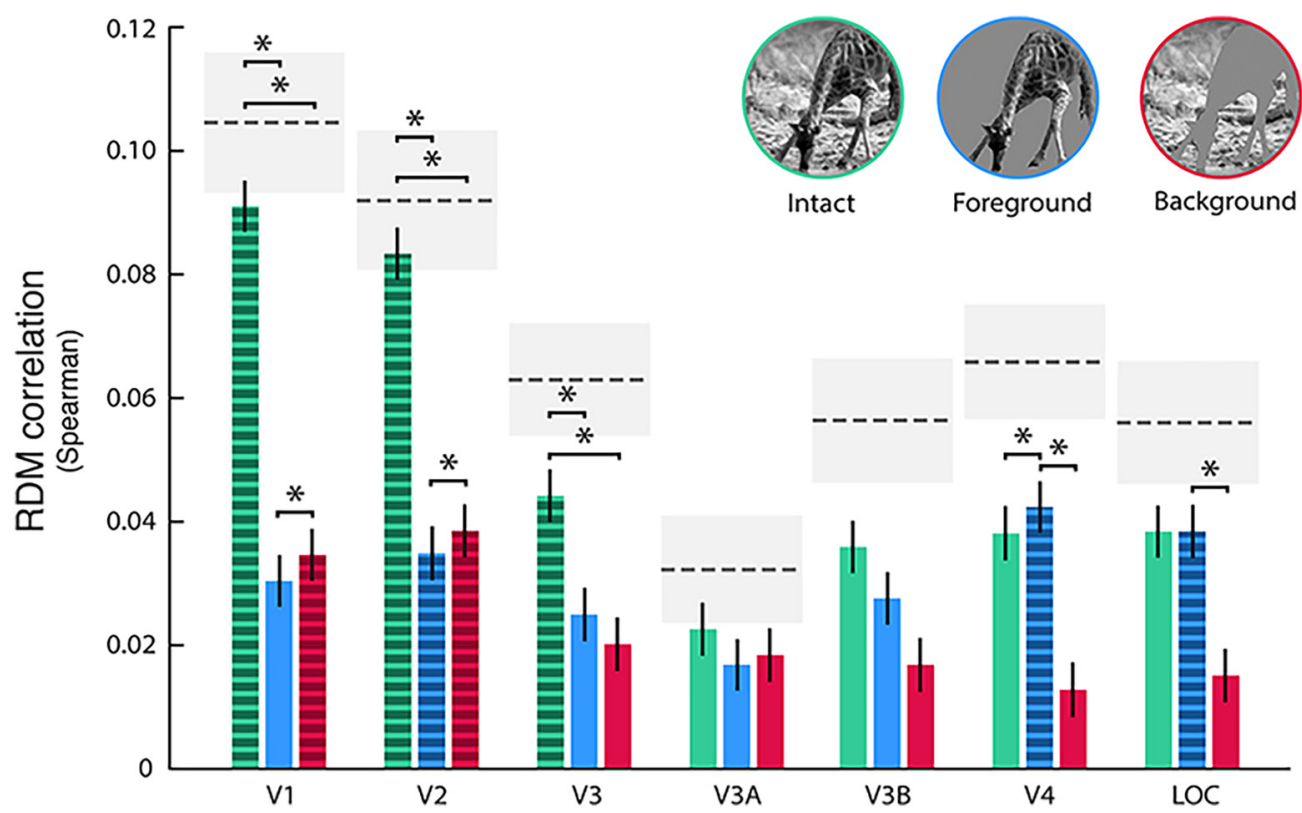

Figure 3. Comparison of intact and behaviorally segmented images. The graphs show the correlation between the intact (green) and segmented versions (blue: isolated foreground; red: isolated background) of the images and brain RDMs ( $n=55611)$. Dashed bars stand for significant correlations as resulting from the permutation test ( $p<0.05$, Bonferroni corrected; 1000 iterations). Asterisks indicate significant differences between correlation values $(p<0.05$, Bonferroni corrected). Error bars represent the SE estimated with bootstrapping. Dashed lines represent the SNR estimate for each ROI, while gray shaded regions indicate its SE.

correlation given the noise of the data), thus confirming the validity of the fixed model employed (Wu et al., 2006).

\section{Foreground is enhanced in all the tested regions}

We tested whether the behavioral foreground segmentation from BSD represented a better predictor of RDMs derived from $\mathrm{fMRI}$ activity, as compared to alternate configurations obtained by shuffling the segmentation patterns across stimuli (Fig. 2A). The correct foreground configuration yielded a significantly higher correlation as compared to the examples from the shuffled dataset (i.e., a null distribution obtained with a permutation test; Table 2), thus suggesting that the enhancement of foreground-related information occurs during passive perception of natural stimuli in all the tested ROls (V1: $p=$ 0.006; V2: $p<0.001$; V3: $p=0.014$; V3A: $p=0.002$; V3B: $p=0.005 ;$ V4: $p<0.001$; LOC: $p<0.001$.

In addition, this analysis rules out two potential confounding effects. One related to a fovea-to-periphery bias in our image set. In fact, as already observed in literature, natural images are typically characterized by objects located at the center of the scene, see for instance the object location bias represented in Alexe et al. (2010; their Fig 3B). However, since the spatial distribution and number of pixels were kept constant at each permutation step, we replicated the same fovea-to-periphery bias in the null

Table 1. Comparison of intact and behaviorally segmented images

\begin{tabular}{|c|c|c|c|c|c|c|}
\hline \multirow[t]{2}{*}{$\mathrm{ROI}$} & \multicolumn{2}{|c|}{ Intact } & \multicolumn{2}{|c|}{ Foreground } & \multicolumn{2}{|c|}{ Background } \\
\hline & Spearman's $\rho$ & $p$ value & Spearman's $\rho$ & $p$ value & Spearman's $\rho$ & $p$ value \\
\hline V1 & $0.091 \pm 0.008$ & $<0.001^{*}$ & $0.03 \pm 0.008$ & 0.006 & $0.035 \pm 0.008$ & $<0.001^{*}$ \\
\hline V2 & $0.084 \pm 0.005$ & $<0.001^{*}$ & $0.035 \pm 0.007$ & $<0.001^{*}$ & $0.039 \pm 0.004$ & $<0.001^{*}$ \\
\hline V3 & $0.044 \pm 0.007$ & $<0.001^{*}$ & $0.025 \pm 0.008$ & 0.08 & $0.02 \pm 0.007$ & 0.29 \\
\hline V3A & $0.023 \pm 0.004$ & 0.34 & $0.017 \pm 0.005$ & 0.256 & $0.018 \pm 0.007$ & 0.127 \\
\hline V3B & $0.036 \pm 0.009$ & 0.017 & $0.028 \pm 0.006$ & 0.02 & $0.017 \pm 0.009$ & 0.186 \\
\hline V4 & $0.038 \pm 0.015$ & 0.027 & $0.043 \pm 0.006$ & $<0.001^{*}$ & $0.013 \pm 0.007$ & 0.915 \\
\hline LOC & $0.038 \pm 0.008$ & 0.015 & $0.038 \pm 0.012$ & $<0.001^{*}$ & $0.015 \pm 0.009$ & 0.543 \\
\hline
\end{tabular}

${ }^{*} p<0.05$ Bonferroni corrected.

Table 2. Statistical analysis

\begin{tabular}{llll}
\hline & Data structure & Type of test & Power \\
a & Single correlation values & Nonparametric permutation test & $p<0.05$ Bonferroni corrected \\
b & Single correlation values & Nonparametric permutation test & $p<0.05$ \\
c & Single correlation values & Nonparametric permutation test & $p<0.05$ Bonferroni corrected
\end{tabular}


distribution. The other confound was related to potential differences in contrast between foreground and background. To account for this, in the permutation test, we matched the RMS contrast of each random segmentation to that of the ground-truth segmentation obtained from BSD. Overall, these control procedures minimize the chance that the observed enhancement is driven by location, size, or contrast of the foreground.

\section{Background suppression occurs in higher cortical areas}

As the correlation between the background RDM and RDM derived from $\mathrm{FMRl}$ activity is significant in V1 and V2 only (Fig. 3), we hypothesized that background-related information is suppressed in "higher" visual cortices. Notably, Poort et al. (2016) described background suppression as a different, but associated, phenomenon with respect to foreground enhancement. Thus, to better characterize where and how background suppression occurs in humans attending to natural images, a further analysis was performed by parametrically filtering out the background of each image, varying its contrast or spatial frequencies (low- and high-pass filtering; Fig. 2C). As the correlation between the representational model of V3A, V3B, and those derived from intact, isolated foreground, and isolated background images is not significant $(p>$ 0.05 Bonferroni corrected), these ROls were not further investigated.

When comparing the correlation value of the intact version of the stimuli and the correlation value of each background filtering step, we found that V1, V2, and V3 show a progressive decay, indicating that the background is actually processed by these regions ( $p<0.05$, Bonferroni corrected). On the other hand, in V4 and LOC, filtering the background produces significantly higher correlations $(p<0.05$ Bonferroni corrected), thus indicating that background information is not different from noise (Fig. 4). These findings suggest that background suppression is actually performed by higher cortical areas, as also depicted in correlation images (Fig. 5).

Of note, to validate the proposed method, we performed a simulation of the fMRI experiment using a fully connected layer of a pretrained convolutional neural network (AlexNet fc6; Krizhevsky et al., 2012). The RDM correlation between each contrast filtering step and the representational geometry from the net (i.e., responses to intact images) was computed as in the fMRI analyses (i.e., fixed model). Then, we assessed ground truth computation of the net by showing it the images at each filtering level, thus checking its sensitivity to background manipulation. Results (data not shown) demonstrate that our pre-filtering modeling approach correctly reveals the ground-truth computation of the net.

\section{Discussion}

In the present study, we illustrated how the manipulation of low-level properties of natural images, and the following correlation with patterns of brain responses during passive viewing of the intact stimuli, could disclose the behavior of different regions along the visual pathway.
Employing this pre-filtering modeling approach, we tested whether scene segmentation is an automatic process that occurs during passive perception in naturalistic conditions, even when individuals are not required to perform any particular tasks, or to focus on any specific aspect of images. Here, we were able to collect three different pieces of evidence confirming our hypothesis on the mechanisms involved in scene segmentation.

First, by using RSA, we demonstrated that representational models built from $\mathrm{fMRI}$ patterns show a significant correlation with isolated foreground in V2, V4, and LOC, while a significant correlation with isolated background is achieved in V1 and V2 only.

Second, our analyses specifically found that foreground enhancement is present in all the selected visual ROls, and that this effect is driven neither by the foreground contrast, nor by its size or location in the visual field. Thus, indirect evidence of figure-ground modulation of natural images could be retrieved in the activity of multiple areas of the visual processing stream (Roelfsema, 2006; Roelfsema and de Lange, 2016). This is consistent with a recent study, which reported that border-ownership of natural images cannot be solved by single cells but requires a population of cells in monkey V2 and V3 (Hesse and Tsao, 2016).

Finally, a proof of segmentation can be represented by the significant suppression of background-related information in V4 and LOC. On the contrary, earlier regions across the visual stream, from $\mathrm{V} 1$ to $\mathrm{V} 3$, have a uniform representation of the whole image, as evident at first glance in the obtained correlation images (Fig. 5). Overall these results further support the idea that foreground enhancement and background suppression are distinct, but associated, processes involved in scene segmentation of natural images.

\section{Foreground segmentation as a proxy for shape processing}

Of note, our proposed pre-filtering modeling approach produces a visual representation (i.e., correlation image) of how information is selectively coded by a specific population of interest (e.g., LOC). Further interpretations on the obtained visual representation may result more empirical and, similarly to other computational neuroimaging methods (e.g., Inverted Encoding Models; Liu et al., 2018), should be grounded on previous neurophysiological knowledge. For instance, the correlation image of LOC could be interpreted as resulting from two alternative mechanisms: LOC could preferentially process the foreground as a whole, while suppressing the background, or it could act as a "feature detector," whose neurons are selectively tuned toward a single visual attribute (e.g., the whiskers of a cat), without actively performing any suppression. Either way, what our method clearly reveals is that LOC is selective for object texture and shape properties and is unaffected by background-related information. At the same time, previous knowledge suggests that an active process, rather than a passive feature-matching mechanism, determines the observed results (Roelfsema and De Lange, 2016). 

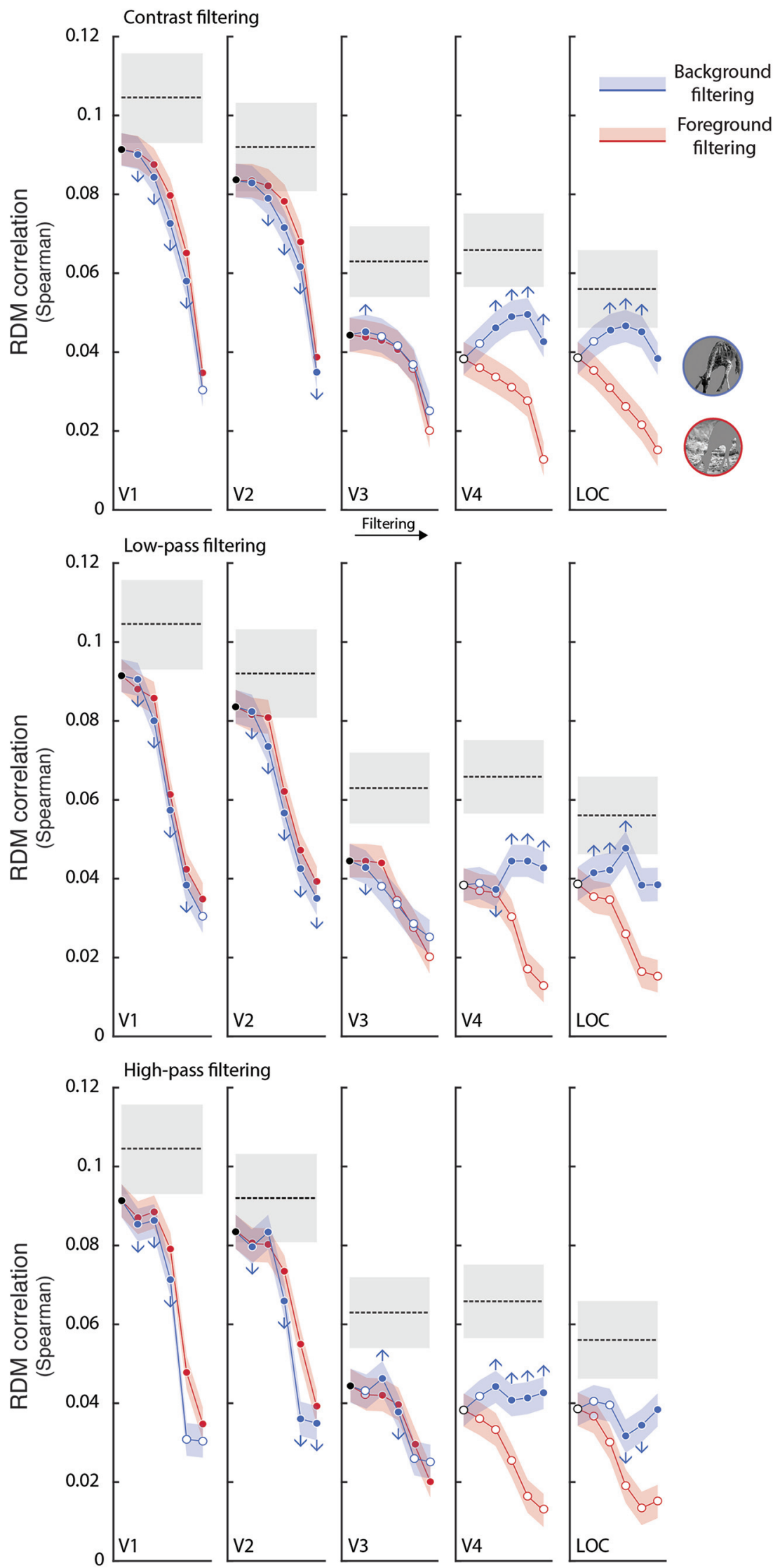

Figure 4. Background suppression in the human visual system. Correlation between brain activity and contrast, low- and high-pass filtering applied to the background (blue) and, as a control, to the foreground (red). Filled dots mark significant correlations $(p<0.05$, 
continued

Bonferroni corrected) while colored shaded areas represent the SE estimates. Dashed lines represent the SNR estimate for each ROI, while gray shaded regions indicate its SE. Arrows stand for significant differences $(p<0.05$, Bonferroni corrected) between each filtering step and correlation values for the intact version (up: background suppression; down: progressive decay). Results show that for early regions $(\mathrm{V} 1-\mathrm{V} 3)$ background-related information is relevant, since the correlation significantly decays due to filtering $(p<$ 0.05 , Bonferroni corrected); on the other hand, V4 and LOC show an opposite effect, suggesting that background is suppressed in those regions.

Furthermore, the observed behavior of V4 and LOC is consistent with several investigations on shape features selectivity in these regions, and in their homologues in monkey (Carlson et al., 2011; Hung et al., 2012; Lescroart and Biederman, 2013; Vernon et al., 2016). In fact, the extraction of shape properties requires segmentation (Lee et al., 1998), and presumably occurs in brain regions where background is already suppressed. As mentioned before, correlation images reconstructed from V4 and LOC are characterized by a strong background suppression, while the foreground is preserved. This is consistent with a previous neuropsychological observation: a bilateral lesion within area V4 led to longer response times in identifying overlapping figures (Leek et al., 2012). Hence, this region resulted to be crucial for accessing foregroundrelated computations, and presumably plays a role in matching the segmented image with stored semantic content in figure recognition. In accordance with this, a recent hypothesis suggests the role of V4 in high-level visual functions, such as features integration or contour completion (Roe et al., 2012).

The preserved spatial resolution of foreground descriptive features (i.e., texture) in V4 and LOC (as shown in Fig. 5) represents an additional noteworthy aspect that arises from our data. The progression from V1 toward higherlevel regions of the cortical visual pathway is associated with a relative increase in receptive fields size (Gattass et al., 1981, 1987, 1988; Dumoulin and Wandell, 2008; Freeman and Simoncelli, 2011; Kay et al., 2015). However, it should be kept in mind that regions such as V4 demonstrate a complete representation of the contralateral visual hemifield, rather than selective responses to stimuli located above or below the horizontal meridian (Wandell and Winawer, 2011). The evidence that the foreground portion of correlation images maintains finegrained details in V4 and LOC seems to contrast a popular view according to which these regions are more tuned to object shape (i.e., silhouettes), instead of being selective for the internal configuration of images (Malach et al., 1995; Grill-Spector et al., 1998; Moore and Engel, 2001; Stanley and Rubin, 2003). However, it has been shown that foveal and peri-foveal receptive fields of V4 do accommodate fine details of the visual field (Freeman and Simoncelli, 2011) and that the topographic representation of the central portion of this area is based on a direct sampling of the primary visual cortex retinotopic map (Motter, 2009). Therefore, given the fovea-to-periphery bias found in our stimuli and in natural images, it is reasonable that an intact configuration of the foreground may be more tied to the activity of these brain regions, and that a richer representation of the salient part may overcome simplistic models of objects shape (e.g., silhouettes). Our results are also consistent with a recent study on monkeys that demonstrates the role of V4 in texture perception (Okazawa et al., 2015).

Moreover, it is well known that selective attention represents one of the cognitive mechanisms supporting figure segmentation (Qiu et al., 2007; Poort et al., 2012), as suggested, for instance, by bistable perception phenomena (Sterzer et al., 2009), or by various neuropsychological tests (De Renzi et al., 1969; Bisiach et al., 1976). In the present experiment, participants were asked to simply gaze a central fixation point without performing any overt or covert tasks related to the presented image. Nonetheless, we found evidence of a clear background suppression and foreground enhancement, suggesting that scene segmentation is mediated by an automatic process that may be driven either by bottom-up (e.g., low-level properties of the foreground configuration), or top-down (e.g., semantic knowledge) attentional mechanisms. Neurophysiological studies suggest that segmentation is more likely a bottom-up process, as border-ownership assignment occurs as early as $70 \mathrm{~ms}$ (Williford and von der Heydt, 2016), followed by later region-filling mechanisms (i.e., enhancement and suppression; Self et al., 2013). A limit of our study is that we cannot provide any further

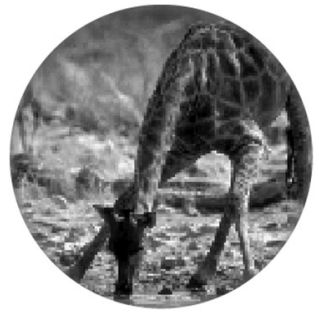

V1

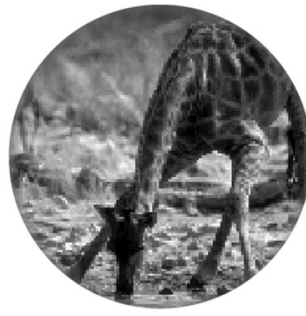

V2

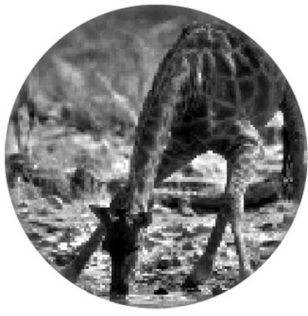

V3

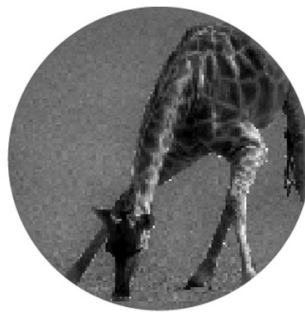

V4

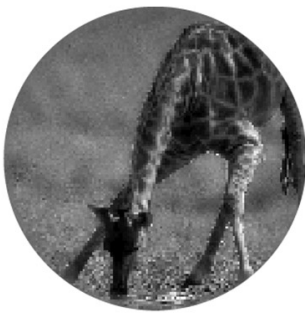

LOC

Figure 5. Correlation images. To visually represent these results, we combined the different filtering procedures (contrast, low- and high-pass filtering) of the step showing the highest correlation with the representational model from each ROI. 
information related to these mechanisms and their temporal dynamics, given the limited temporal resolution of fMRI and the passive stimulation task. However, a recent study (Neri, 2017) investigated behavioral and electrophysiological responses to BSD images, intact or manipulated in several different ways, including spatial frequencies filtering and warping, in subjects who were asked to reconstruct a corrupted image region. Results showed that reconstruction of patches elicits enhanced responses when masking targeted the behaviorally segmented contours, rather than the contrast energy of the images. Moreover, this effect occurs earlier than $100 \mathrm{~ms}$ and is not altered by semantic processing or spatial attention.

\section{Facing the challenge of explicit modeling in visual neuroscience}

One of the major goals of visual neuroscience is to predict brain responses in ecological conditions (Felsen and Dan, 2005). In this sense, the standard approach in investigating visual processing implies testing the correlation of brain responses from a wide range of natural stimuli with features extracted by different alternative computational models. This approach facilitates the comparison between performances of competing models and could ultimately lead to the definition of a fully computable model of brain activity. However, the development of explicit computational models for many visual phenomena in ecological conditions is difficult. Indeed, many current theories, especially those concerning mid-level processing, have been hardly tested with natural images, as testified by the extensive use of artificial stimuli (Carandini et al., 2005; Wu et al., 2006). As a matter of fact, it is often impossible both to extract and to control for relevant features in natural images, and thus, there is no way to compute a predicted response from complex stimuli.

Moreover, even if computer vision is a major source of computational models and feature extractors, often its objectives hardly overlap with those of visual neuroscience. Computer scientists are mainly interested in solving single, distinct tasks (e.g., segmentation, recognition, etc.), while, from the neuroscientific side, the visual system is considered as a general-purpose system that could retune itself to accomplish different goals (Medathati et al., 2016). Consequently, while computer science typically employs solutions that rely only seldom on previous neuroscientific knowledge, and its goal is to maximize task accuracy (e.g., with deep learning), visual neuroscience somehow lacks of solid computational models and formal explanations, ending up with several arbitrary assumptions in modeling, especially for mid-level vision processing, such as scene segmentation or shape features extraction (for a definition, see Kubilius et al., 2014).

In light of all this, we believe that the manipulation of a wide set of natural images, and the computation of a fixed model based on low-level features, can offer a simple and biologically plausible tool to investigate brain activity related to higher-order computations, and that representational models offer an easily accountable link between brain activity and continuous stimuli descriptions (Nili et al., 2014). In fact, the results of this exploratory approach can be depicted and are as intuitive as descriptions obtained through formal modeling (Fig. 5), highlighting interpretable differences rather than data predictions.

Moreover, our study indicates that the sensitivity of representational models built on $\mathrm{AMRI}$ patterns can represent an adequate tool to investigate complex phenomena through the richness of natural stimuli. Representational models fit this purpose: even if are summary statistics obtained from the dissimilarities between actual brain activity, they are independent from a priori assumptions on anatomic relationships between brian regions, or on correspondences between voxels and units of computational models, as in the case of voxelwise encoding or decoding (Kriegeskorte and Kievit, 2013).

\section{References}

Alexe B, Deselaers T, Ferrari V (2010) ClassCut for unsupervised class segmentation. Lect Notes Comput Sci 6315:380-393.

Arbeláez P, Maire M, Fowlkes C, Malik J (2011) Contour detection and hierarchical image segmentation. IEEE Trans Pattern Anal Mach Intell 33:898-916. CrossRef Medline

Biederman I (1987) Recognition-by-components - a theory of human image understanding. Psychol Rev 94:115-147. Medline

Bisiach E, Capitani E, Nichelli P, Spinnler H (1976) Recognition of overlapping patterns and focal hemisphere damage. Neuropsychologia 14:375-379. Medline

Bosch A, Zisserman A, Munoz X (2007) Representing shape with a spatial pyramid kernel, pp 401-408. ACM. https://dl.acm.org/ citation. $\mathrm{cfm}$ ? id $=1282340$

Carandini M, Demb JB, Mante V, Tolhurst DJ, Dan Y, Olshausen BA, Gallant JL, Rust NC (2005) Do we know what the early visual system does?. J Neurosci 25:10577-10597. CrossRef Medline

Carlson ET, Rasquinha RJ, Zhang K, Connor CE (2011) A sparse object coding scheme in area V4. Curr Biol 21:288-293. CrossRef Medline

David SV, Vinje WE, Gallant JL (2004) Natural stimulus statistics alter the receptive field structure of $v 1$ neurons. J Neurosci 24:69917006. CrossRef Medline

De Renzi E, Scotti G, Spinnler H (1969) Perceptual and associative disorders of visual recognition. Relationship to the side of the cerebral lesion. Neurology 19:634-642. CrossRef

Dumoulin SO, Wandell BA (2008) Population receptive field estimates in human visual cortex. Neuroimage 39:647-660. CrossRef Medline

Efron B, Tibshirani R (1993) An introduction to the bootstrap. New York: Chapman \& Hall.

Felsen G, Dan Y (2005) A natural approach to studying vision. Nat Neurosci 8:1643-1646. CrossRef Medline

Fowlkes CC, Martin DR, Malik J (2007) Local figure-ground cues are valid for natural images. J Vis 7:2. CrossRef Medline

Freeman J, Simoncelli EP (2011) Metamers of the ventral stream. Nat Neurosci 14:1195-1201. CrossRef Medline

Gattass R, Gross CG, Sandell JH (1981) Visual topography of V2 in the macaque. J Comp Neur 201:519-539. CrossRef Medline

Gattass R, Sousa AP, Rosa MG (1987) Visual topography of V1 in the Cebus monkey. J Comp Neur 259:529-548. CrossRef Medline

Gattass R, Sousa AP, Gross CG (1988) Visuotopic organization and extent of V3 and V4 of the macaque. J Neurosci 8:1831-1845. Medline

Grill-Spector K, Kushnir T, Edelman S, Itzchak Y, Malach R (1998) Cue-invariant activation in object-related areas of the human occipital lobe. Neuron 21:191-202. Medline

Handjaras G, Leo A, Cecchetti L, Papale P, Lenci A, Marotta G, Pietrini P, Ricciardi E (2017) Modality-independent encoding of individual concepts in the left parietal cortex. Neuropsychologia 105:39-49. CrossRef Medline 
He K, Gkioxari G, Dollár P, Girshick R (2017) Mask R-CNN. arXiv preprint arXiv:170306870.

Hesse JK, Tsao DY (2016) Consistency of border-ownership cells across artificial stimuli, natural stimuli, and stimuli with ambiguous contours. J Neurosci 36:11338-11349. CrossRef Medline

Hung CC, Carlson ET, Connor CE (2012) Medial axis shape coding in macaque inferotemporal cortex. Neuron 74:1099-1113. CrossRef Medline

Huth AG, Nishimoto S, Vu AT, Gallant JL (2012) A continuous semantic space describes the representation of thousands of object and action categories across the human brain. Neuron 76:12101224. CrossRef Medline

Kastner S, De Weerd P, Ungerleider LG (2000) Texture segregation in the human visual cortex: a functional MRI study. J Neurophysiol 83:2453-2457. CrossRef Medline

Kay KN, Naselaris T, Gallant JL (2011) fMRI of human visual areas in response to natural images. Available at https://crcns.org/datasets/vc/vim-1.

Kay KN, Naselaris T, Prenger RJ, Gallant JL (2008) Identifying natural images from human brain activity. Nature 452:352-355. CrossRef Medline

Kay KN, Weiner KS, Grill-Spector K (2015) Attention reduces spatial uncertainty in human ventral temporal cortex. Curr Biol 25:595600. CrossRef Medline

Khaligh-Razavi S-M (2014) What you need to know about the stateof-the-art computational models of object-vision: a tour through the models. arXiv preprint arXiv: 14072776 .

Khaligh-Razavi SM, Kriegeskorte N (2014) Deep supervised, but not unsupervised, models may explain IT cortical representation. PLoS Comput Biol 10:e1003915. CrossRef

Kok P, de Lange FP (2014) Shape perception simultaneously up- and downregulates neural activity in the primary visual cortex. Curr Biol 24:1531-1535. CrossRef Medline

Kriegeskorte N, Kievit RA (2013) Representational geometry: integrating cognition, computation, and the brain. Trends Cogn Sci 17:401-412. CrossRef Medline

Kriegeskorte N, Mur M, Ruff DA, Kiani R, Bodurka J, Esteky H, Tanaka K, Bandettini PA (2008) Matching categorical object representations in inferior temporal cortex of man and monkey. Neuron 60:1126-1141. CrossRef Medline

Krizhevsky A, Sutskever I, Hinton GE (2012) Imagenet classification with deep convolutional neural networks, pp 1097-1105. Advances in neural information processing systems. http:// papers.nips.cc/paper/4824-imagenet-classification-with-deepconvolutional-neural-networks.

Kubilius J, Wagemans J, Op de Beeck HP (2014) A conceptual framework of computations in mid-level vision. Front Comput Neurosci 8:158. CrossRef Medline

Kubilius J, Bracci S, Op de Beeck HP (2016) Deep neural networks as a computational model for human shape sensitivity. PLoS Comput Biol 12:e1004896. CrossRef Medline

Lamme VA (1995) The neurophysiology of figure-ground segregation in primary visual cortex. J Neurosci 15:1605-1615. Medline

Lamme VA, Rodriguez-Rodriguez V, Spekreijse H (1999) Separate processing dynamics for texture elements, boundaries and surfaces in primary visual cortex of the macaque monkey. Cereb Cortex 9:406-413. Medline

Lazebnik S, Schmid C, Ponce J (2006) Beyond bags of features: spatial pyramid matching for recognizing natural scene categories. Proceedings of the IEEE Computer Society Conference on Computer Vision and Pattern Recognition (CVPR '06); June 2006; pp 2169-2178.

Lee TS, Mumford D, Romero R, Lamme VA (1998) The role of the primary visual cortex in higher level vision. Vision Res 38:24292454. Medline

Leek EC, d'Avossa G, Tainturier MJ, Roberts DJ, Yuen SL, Hu M, Rafal R (2012) Impaired integration of object knowledge and visual input in a case of ventral simultanagnosia with bilateral damage to area V4. Cogn Neuropsychol 29:569-583. CrossRef
Lescroart M, Agrawal P, Gallant J (2016) Both convolutional neural networks and voxel-wise encoding models of brain activity derived from ConvNets represent boundary-and surface-related features. $J$ Vis 16:756-756. CrossRef

Lescroart MD, Biederman I (2013) Cortical representation of medial axis structure. Cereb Cortex 23:629-637. CrossRef Medline

Liu T, Cable D, Gardner JL (2018) Inverted encoding models of human population response conflate noise and neural tuning width. J Neurosci 38:398-408. CrossRef Medline

Malach R, Reppas JB, Benson RR, Kwong KK, Jiang $H$, Kennedy WA, Ledden PJ, Brady TJ, Rosen BR, Tootell RB (1995) Objectrelated activity revealed by functional magnetic resonance imaging in human occipital cortex. Proc Natl Acad Sci USA 92:8135-8139. Medline

Marr D (1982) Vision: a computational investigation into the human representation and processing of visual information. San Francisco: W.H. Freeman.

Medathati NVK, Neumann H, Masson GS, Kornprobst P (2016) Bio-inspired computer vision: towards a synergistic approach of artificial and biological vision. Comput Vis Image Underst 150:130. CrossRef

Miyawaki Y, Uchida H, Yamashita O, Sato M-a, Morito Y, Tanabe HC, Sadato N, Kamitani Y (2008) Visual image reconstruction from human brain activity using a combination of multiscale local image decoders. Neuron 60:915-929. CrossRef Medline

Moore C, Engel SA (2001) Neural response to perception of volume in the lateral occipital complex. Neuron 29:277-286. Medline

Motter BC (2009) Central V4 receptive fields are scaled by the V1 cortical magnification and correspond to a constant-sized sampling of the V1 surface. J Neurosci 29:5749-5757. CrossRef

Naselaris T, Kay KN, Nishimoto S, Gallant JL (2011) Encoding and decoding in fMRI. Neuroimage 56:400-410. CrossRef Medline

Neri P (2017) Object segmentation controls image reconstruction from natural scenes. PLoS Biol 15:e1002611. CrossRef Medline

Nili H, Wingfield C, Walther A, Su L, Marslen-Wilson W, Kriegeskorte N (2014) A toolbox for representational similarity analysis. PLoS Comput Biol 10:e1003553. CrossRef Medline

Nishimoto S, Vu AT, Naselaris T, Benjamini Y, Yu B, Gallant JL (2011) Reconstructing visual experiences from brain activity evoked by natural movies. Curr Biol 21:1641-1646. CrossRef Medline

Ojala T, Pietikäinen M, Mäenpää T (2001) A generalized local binary pattern operator for multiresolution gray scale and rotation invariant texture classification, pp 399-408. Heidelberg: Springer.

Okazawa G, Tajima S, Komatsu H (2015) Image statistics underlying natural texture selectivity of neurons in macaque V4. Proc Natl Acad Sci USA 112:E351-E360. CrossRef Medline

Oliva A, Torralba A (2001) Modeling the shape of the scene: a holistic representation of the spatial envelope. Int $\mathrm{J}$ Comput Vis 42:145175. CrossRef

Poort J, Raudies F, Wannig A, Lamme VA, Neumann H, Roelfsema PR (2012) The role of attention in figure-ground segregation in areas V1 and V4 of the visual cortex. Neuron 75:143-156. CrossRef Medline

Poort J, Self MW, van Vugt B, Malkki H, Roelfsema PR (2016) Texture segregation causes early figure enhancement and later ground suppression in areas V1 and V4 of visual cortex. Cereb Cortex 26:3964-3976. CrossRef Medline

Qiu FT, Sugihara T, von der Heydt R (2007) Figure-ground mechanisms provide structure for selective attention. Nat Neurosci 10: 1492-1499. CrossRef Medline

Roe AW, Chelazzi L, Connor CE, Conway BR, Fujita I, Gallant JL, Lu H, Vanduffel W (2012) Toward a unified theory of visual area V4. Neuron 74:12-29. CrossRef Medline

Roelfsema PR (2006) Cortical algorithms for perceptual grouping. Annu Rev Neurosci 29:203-227. CrossRef Medline

Roelfsema PR, de Lange FP (2016) Early visual cortex as a multiscale cognitive blackboard. Annu Rev Vis Sci 2:131-151. CrossRef Medline

Scholte HS, Jolij J, Fahrenfort JJ, Lamme VA (2008) Feedforward and recurrent processing in scene segmentation: electroence- 
phalography and functional magnetic resonance imaging. J Cogn Neurosci 20:2097-2109. CrossRef Medline

Schreiber K, Krekelberg B (2013) The statistical analysis of multivoxel patterns in functional imaging. PLoS One 8:e69328. CrossRef Medline

Self MW, van Kerkoerle T, Supèr H, Roelfsema PR (2013) Distinct roles of the cortical layers of area $\mathrm{V} 1$ in figure-ground segregation. Curr Biol 23:2121-2129. CrossRef Medline

Stanley DA, Rubin N (2003) fMRI activation in response to illusory contours and salient regions in the human lateral occipital complex. Neuron 37:323-331. Medline

Stanley GB, Li FF, Dan Y (1999) Reconstruction of natural scenes from ensemble responses in the lateral geniculate nucleus. J Neurosci 19:8036-8042. Medline

Sterzer P, Kleinschmidt A, Rees G (2009) The neural bases of multistable perception. Trends Cogn Sci 13:310-318. CrossRef Medline
Thirion B, Duchesnay E, Hubbard E, Dubois J, Poline JB, Lebihan D, Dehaene S (2006) Inverse retinotopy: inferring the visual content of images from brain activation patterns. Neuroimage 33:1104-1116. CrossRef Medline

Vernon RJ, Gouws AD, Lawrence SJ, Wade AR, Morland AB (2016) Multivariate patterns in the human object-processing pathway reveal a shift from retinotopic to shape curvature representations in lateral occipital areas, LO-1 and LO-2. J Neurosci 36:57635774. CrossRef Medline

Wandell BA, Winawer $\mathrm{J}$ (2011) Imaging retinotopic maps in the human brain. Vision Res 51:718-737. CrossRef Medline

Williford JR, von der Heydt R (2016) Figure-ground organization in visual cortex for natural scenes. eNeuro 3.

Wu MC, David SV, Gallant JL (2006) Complete functional characterization of sensory neurons by system identification. Annu Rev Neurosci 29:477-505. CrossRef Medline 